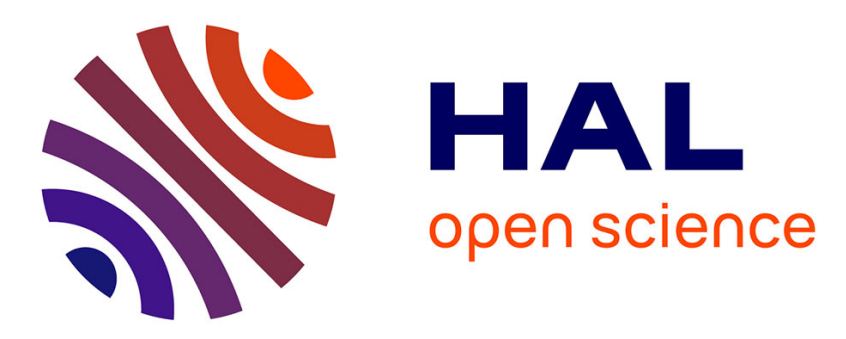

\title{
A new structured adjustment approach for demand forecasting
}

François Marmier, Maria Gonzales-Blanch, Naoufel Cheikhrouhou

\section{To cite this version:}

François Marmier, Maria Gonzales-Blanch, Naoufel Cheikhrouhou. A new structured adjustment approach for demand forecasting. 39th International Conference on Computers \& Industrial Engineering (CIE39), Jul 2009, Troyes, France. p.785-790, 10.1109/ICCIE.2009.5223733 . hal-01112298

\section{HAL Id: hal-01112298 \\ https://hal.science/hal-01112298}

Submitted on 2 Feb 2015

HAL is a multi-disciplinary open access archive for the deposit and dissemination of scientific research documents, whether they are published or not. The documents may come from teaching and research institutions in France or abroad, or from public or private research centers.
L'archive ouverte pluridisciplinaire HAL, est destinée au dépôt et à la diffusion de documents scientifiques de niveau recherche, publiés ou non, émanant des établissements d'enseignement et de recherche français ou étrangers, des laboratoires publics ou privés. 


\title{
A new structured adjustment approach for demand forecasting
}

\author{
François Marmier $^{1,2}$, Maria Gonzales-Blanch ${ }^{1}$, Naoufel Cheikhrouhou ${ }^{1}$ \\ ${ }^{1}$ Ecole Polytechnique Fédérale de Lausanne (EPFL), Laboratory for Production Management and \\ Processes, CH-1015, Lausanne, Switzerland (naoufel.cheikhrouhou@epfl.ch) \\ ${ }^{2}$ Université de Toulouse, MINES ALBI - CGI, 81013, Albi, France (Francois.marmier@mines-albi.fr)
}

\begin{abstract}
Demand forecasting consists of using data of the past demand to obtain an approximation of the future demand. Mathematical approaches can lead to reliable forecasts in deterministic context, extrapolating regular patterns in series. However unpredictable events that do not appear in the historical data can make the forecasts obsolete. As forecasters have a partial knowledge of the context and probable future events (such as strikes, promotions), this work investigates structuring the implicit as well as the explicit knowledge in order to be easily and fully integrated into final forecasts. This paper presents a judgmental-based approach in forecasting where mathematical forecasts are considered as a basis and the structured knowledge of the experts is provided to adjust the initial forecasts. This is achieved using the identification of four factors characterizing specific events that could not have been considered in the initial forecasts. The validation of this approach has been conducted on 2 industrial case studies, a plastic bag manufacturer and a distributor on the food market. The results show that structuring the expert knowledge could lead not only to high improvements of forecasts accuracy but also to a better initial data cleaning and outlier identifications.
\end{abstract}

Keywords: Forecasting, Demand planning, Judgmental forecasting, Judgmental factors, Time series

\section{INTRODUCTION}

Demand planning and forecasting using time series consists of using historical data of the past demand to obtain an approximation of the future demand. Many different ways of developing forecasts have been studied or proposed, like the mathematical and statistical methods or probabilistic approaches to model random variables [1]. Although mathematical approaches can lead to reliable forecasts in some contexts, extrapolating regular patterns in series to unpredictable events can render the forecasts obsolete. On the other hand, forecasters have for asset their partial knowledge of the context and of potential external stunner. This knowledge is not available within the statistical methods as it corresponds to particular events, such as personnel strike or limited promotions. Nowadays, it has been recognized that judgment is an indispensable component of forecasting [2] and that there still is a need for studies which could help companies to improve their forecast quality using contextual information [3], [4].

The objective of this work is the development of a methodological approach for the improvement of forecasting accuracy by structuring and using expert knowledge. A judgmental adjustment based approach, combining mathematical forecast and judgmental factors, is discussed and its performances are compared to classical mathematical forecasting techniques. The comparison of the forecasting accuracy of the considered approaches is done using error measures. Furthermore, the increasing accuracy of the proposed methods in forecasting is evaluated on the basis of two industrial applications within a plastic bag manufacturer in Spain and a food distributor in France.

In Section 2, an overview of the features of the forecasting techniques over the literature including the judgmental forecasting methods is presented and discussed. Section 3 outlines the details of the proposed judgmental adjustment-based approach and its main features. The performances of the proposed approach are compared in Section 4. Two industrial case studies using the developed approach are presented in section 5 . Finally, Section 6 contains the conclusions of this work and future research directions.

\section{COMBINED \\ JUDGMENTAL-MATHEMATICAL FORECASTING SYSTEMS}

In the literature review [5], four main types of approach integrating human judgment into forecasting methods are defined: the model building approach is a methodology which uses judgment in the selection and development of the quantitative forecast [6]. This approach requires high technical knowledge. The combination of judgmental and mathematical forecasts is a pragmatic approach for integrating the personal analysis of contextual information that may influence the forecasts [7]. It has been shown that it gives good results for time series which have a low variation coefficient [8]. Moreover, both (mathematical and contextual) forecasts have to present similar accuracies to lead to reliable global forecast. The judgmental 
decomposition consists of identifying and analysing the effects of past contextual information in time series before establishing mathematical forecasts. Once the mathematical forecasts are established, future-oriented factors are taken into account and the forecasts are adjusted judgmentally [9]. From the planner point of view, it is possible that decomposition reduces the cognitive workload, but it may be risky and ineffective in some circumstances. In the judgemental adjustment process, the mathematical forecasts are reviewed by a forecaster and then adjusted on the basis of his/her knowledge and experience. It is a common practice in companies and it is the major competing alternative to the combination process for integrating judgment with mathematical forecasts. However, the practice of judgmental adjustment has been criticized because of its informal nature [6]. Using a procedure or a decision support system to structure information is helpful in both decision-making and forecasting. Judgmental adjustments lead to greater improvements in accuracy when the process is structured. The forecasting process is performed sequentially with the quantitative forecasts generated first, and then adjusted based on judgment. One of the advantages of this approach is time reduction, allowing the judgment to rapidly incorporate the latest updated information [4]. Flores et al. [10] compare different approaches of judgemental adjustment; The forecaster ranks the impacts due to different factors to obtain an average adjustment of an ARIMA forecast.

In order to integrate human judgment factors in mathematical models in a structured way, this work proposes a factor-based approach to assist the forecaster to focus selectively on different events and to structure its judgment when adjusting forecasts and enhance the accuracy of forecasts. The approach proposes a classification of event-based factors used in structuring the information. Similar factors are proposed by Lee et al. [11] to determine the reaction of the demand faced to different situations; These factors are used in a casebased reasoning software playing the role of forecasting expert. In our case, the forecaster will be able to structure and communicate his implicit knowledge concerning markets evolution (total volume, number of competitors...), customers (numbers, needs, potential demand...) and contractors (development status, special offers...) by representative factors.

\section{PROPOSED APPROACH}

The global approach consists of integrating the expert judgment in a structured manner as complementary information to mathematical forecasts, established by means of classical mathematical models. Figure 1 describes this idea composed of three steps: first, data are filtered and initial mathematical forecasts are developed. Second, different probable events are identified by experts and the related factor variables are weighted and finally, the mathematical forecasts are adjusted.

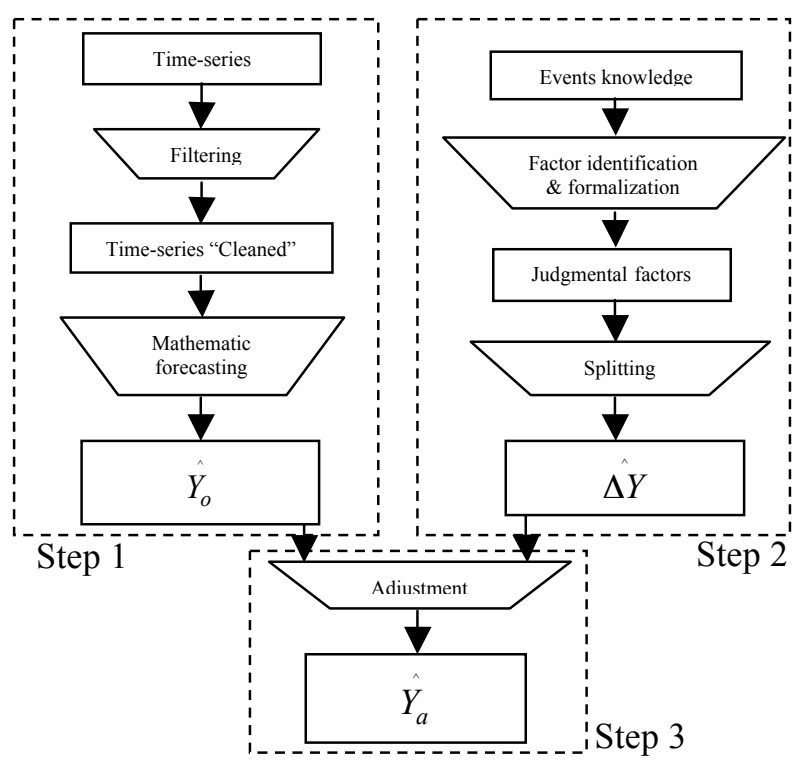

Figure 1. Conceptual scheme of the general method developed

\subsection{First step: data filtering and mathematic forecasting}

This step consists of identifying the outliers and removing the effect of non-periodic events on the data series. The time series is decomposed, taking into account past contextual information. In other words, the data $Y(t-k)$ of the period $t-k$ of the time-series is cleaned (noted $Y o(t-k))$. Historical judgmental events can usually be identified by examining the corresponding historical records. In addition, in order to prevent from the risk of missing or ignoring some historical judgmental events, outliers may be examined and analyzed in detail. Outliers are considered as unusual or abnormal observations in data and are replaced by representative values (f.i. the average of the neighboring values). If the time series presents a strong trend or a seasonal pattern, a confidence interval is evaluated for each year and each time period. Thus, the mathematical forecasts for the given filtered or "cleaned" time-series for period $t$ are

$$
\wedge
$$

$$
Y_{0}(t)=f\left(Y_{0}(t-1), f\left(Y_{0}(t-2), \ldots\right)\right.
$$

\subsection{Second step: Identification and formalization of influencing factors}

A judgmental factor is defined as a "factor that cannot be fully incorporated into the time series models, and thus cannot be effectively identified by the extrapolation of past patterns in the data set" [12]. The attributes or causal variables are the variables that cause changes in the factors. The attributes related to the a factor are: duration, intensity, type, etc. The realization of judgmental factor is considered as a judgmental event. Thus, a judgmental event is accompanied by its impact and may affect the demand during more than one time 
period. Judgmental events can be the realization of different types of judgmental factors. So, a judgmental event impact is split between the relevant judgmental instance periods. The type of judgmental factors may vary from an activity sector to another [11]. The judgmental factors considered in this work consist of 4 types: a- transient factors, b- quantum jump factors, ctransferred impact factors and $\mathrm{d}$ - trend change factors. Each factor is the consequence of a causal variable. It leads the experts to make adjustments of the initial forecasts. Each adjustment, resulting of a judgmental factor is noted $F(i, t)$ where $F$ is the adjustment, $i$ is the total impact and $t$ is the time. The impact $i$ can reach maximal value $\Delta^{\max }$. Then the expert expresses his opinion by weighting $\Delta^{\max }$ with a weight $w$, chosen using the following impact scale:

1- Highest: $80<w<100 \%$.

2- Higher than normal: $60<w<80 \%$.

3- Normal: $40<\mathrm{w}<60 \%$.

4- Lower than normal: $20<w<40 \%$.

5- Lowest: $0<w<20 \%$.

Then : $i=w . \Delta^{\max }$

\section{Transient factors}

Transient factors influence time series data only during the period in which a particular event occurs (such as a strike) (cf. figure 2.a). When the event is over, the effect does not last any longer. The total impact $i$ covers the effective number of impacted periods. So, the time can be decomposed into periodical instances tp. Adjustments that encompass $n$ consecutive periods $p$ are detailed in equation 3 .

$$
F(i, t)=\sum_{p=1}^{n} f\left(i_{p}, t_{p}\right)
$$

where $f$ are the periodical adjustment functions, $i_{p}$ and $t_{p}$ corresponds to the impacted period $p$ with the intensity $i_{p}$. Transient factors render the data be upper or lower than the value of the main trend $\hat{Y}_{0}$. The expert establishes first, the sign of the adjustment (positive or negative) then, the maximal value of the adjustment $\Delta^{\max }$ and finally, following his knowledge of the industrial and commercial context, the expert deduces the weight $w$ in order to determine the impact.

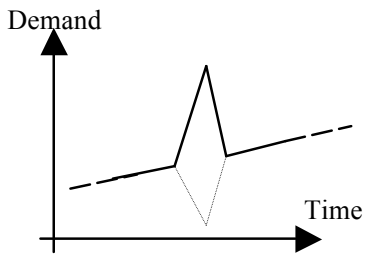

Figure 2.a Transient factor

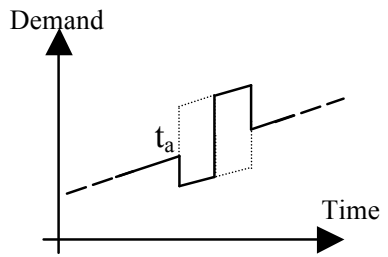

Figure 2.b Transferred impact factor

\section{Transferred impact factors}

The global impact $i$ of this kind of event is transferred from one set of periods $S_{p 1}$ to another one $S_{p 2}$,without changing the global forecasts of the related consecutive $n$ periods. Figure $2 . \mathrm{b}$ presents this factor. This phenomenon happens when the price changes are announced beforehand at time $t_{a}$. In this case, the temporary change in demand due to the expected price change is transferred and compensated in the following time period at time $t_{e}$. Then, $S_{p 1}$ precedes $S_{p 2}$. But if $t_{a}=$ $t_{e}, S_{p 2}$ precedes $S_{p 1}$. Periodical instances that encompass $n$ consecutive periods is computed using equation (4):

$$
F(i, t)=\sum_{p=1}^{n} f\left(i_{p}, t_{p}\right)
$$

Where $i_{p}$ is the proportional impact (positive or negative) on the period $p$. As for the other factors, the expert determine the maximal value $\Delta^{\max }$ subject to be transferred then uses the scale of impact to rate the event and to obtain $i$.

\section{Quantum jump factors}

The quantum jump factor occurs when the effect of a non-repetitive new event is permanent. Figure 3.a presents this factor.

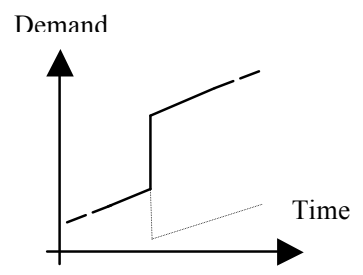

Figure 3.a Quantum jump factor

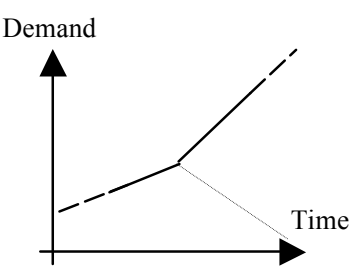

Figure 3.b Trend change factor
The adjustment created by a quantum factor is evaluated and separated in different periodically instances in order to be adjusted to the original mathematical forecast. This adjustment is detailed in the equation 5 where $t_{e}$ is the effective date (the date of the event generating the demand jump)

$$
F(i, t)=i \cdot Q j
$$

Where $Q j$ is a binary variable which means that the Y-intercept, which define the elevation of the demand trend, is impacted with the intensity $i$ at $t_{e}$ :

If $t<t_{e}$ then $Q j=0$

Else $Q j=1$.

The weighting process of the jump factor variables is similar to the weightings of the other factors. After determining the adjustment sign, an expert determines the maximal value of the adjustment $\Delta^{\max }$ and express his /her opinion about the percentage that weights that variable. 


\section{Trend change factors}

Some factors like price variation can modify the demand by a percentage. These factors are considered as Trend change factors as shown in figure 3.b. The expert establishes the sign of the possible trend change and its limit $\Delta^{\max }$. Then he weighs the impact using the impact scale. The trend adjustment is detailed in equation 6 where $t_{e}$ is the effective date of the event generating the demand jump.

$$
F(i, t)=i . t . T c
$$

Where $T c$ is a binary variable which means that the slope of a linear regression of the demand trend is impacted with the intensity $i$ at $t_{e}$ :

If $t<t_{e}$ then $T c=0$

Else $T c=1$.

\subsection{Third step: the adjustment process}

The final adjusted forecast is defined as:

$$
\hat{Y}_{a}(t)=\hat{Y}_{o}(t)+\hat{\Delta} Y^{(t)}
$$

Where $\hat{\Delta} Y^{(t)}$ is the adjustment computed with the judgmental factors established by experts. It corresponds to the total impact on demand of the different event occurring at time $t$. Some factors influenced the demand over a restricted period or a set of periods but others are definitely modifying the demand behaviour. When multiple judgmental factors impact simultaneously the demand, it is necessary to decompose forecast adjustments according to the effects of each individual judgmental event. Then, the global adjustment at time $t$, defined in equation 8 , corresponds to the total $K$ of the different adjustments related to the $K$ factors impacting the demand at time $\mathrm{t}$ :

$$
\hat{\Delta Y}(t)=\sum_{k=1}^{K} F_{k}\left(i_{k}, t\right)
$$

\section{EVALUATION OF ADJUSTED FORECAST QUALITY}

Accuracy measures how well the results of the forecasting model correspond to the actual data. Accuracy plays an important role in evaluating forecasting methods and demand planning. Forecast accuracy is evaluated using different error measures. In general, the error measure used is the one that most closely related to the decision being made. Some commonly used error measures are Mean Absolute Deviation (MAD), Mean Absolute Error (MAE) (cf. equation 9), Mean Percentage Error (MPE), Mean Square Error (MSE) and Root Mean Square Error (RMSE), R2 (or R-squared), Mean Absolute Percentage Error (MAPE) (cf. equation 10).

Armstrong (2001) alleged that R2 (which assesses the pattern of the forecasts relative to that of the actual data), is not particularly useful to forecasters and its use is probably more harmful when using time-series data. Moreover, Armstrong explains why MSE should not be used due to its unreliability in explaining the results. Like MSE, RMSE penalizes errors according to their magnitude. Both MSE and RMSE are not unit-free and therefore comparisons across series are difficult. With MAD there is no way to know if MAD error is large or small with respect to the actual data. With the ME and MPE approach, the real significance of the error can be difficult to evaluate because errors can be annulated by summing negative and positive components. Contrary to $\mathrm{ME}$, the use of MAE makes it possible to take into account both positive and negative errors. With MAE, the total magnitude of error is provided but not the real bias or direction of the error. MAPE measures the deviation as a percentage of actual data with respect to the forecast. Thus, positive and negative errors do not cancel out.

In this paper, MAE and MAPE are used to calculate the forecast errors as they are considered as being the most performing approaches to measure errors.

$$
\text { Mean Absolute Error }(M A E)=\frac{1}{n} \sum_{n}\left|e_{t}\right|
$$

Mean Absolute Percentage Error

$$
(M A P E)=\frac{1}{n} \sum_{t=1}^{n}\left|\frac{Y_{t}-F_{t}}{Y_{t}}\right|
$$

\section{INDUSTRIAL APPLICATION}

\subsection{First case study}

Consider the case of the Company X, a plastic bag manufacturer in the south of Spain. The time series used are composed by the aggregated monthly demand collected over three years (2004-2006) as it can be observed in figure 7 . The aim of this scenario is to plan demand for the year 2007. Company $\mathrm{X}$ has three main customers in this area which are all supermarket chains. An expert analyzes all the coming events and the factors influencing in the plastic bag demand.

The model that gives the smallest MAE and MAPE is $\operatorname{ARIMA}(5,0,4)$. Figure 4 presents the time series and the mathematical forecasts obtained.

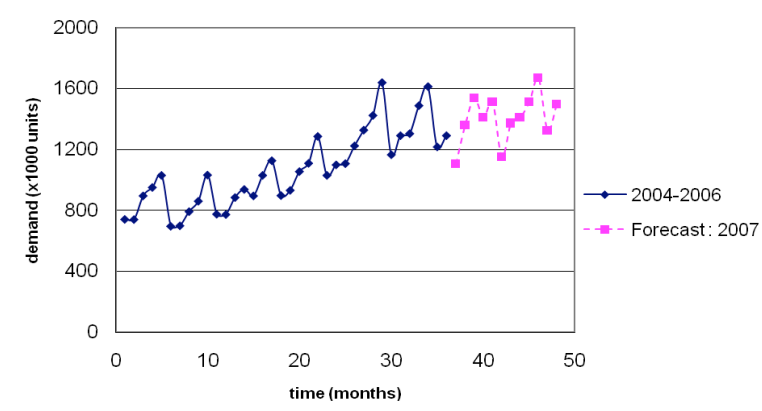

Figure 4. Company X’s demand (2004-2007)

The forecasting expert of company $\mathrm{X}$ identifies and classifies different factors for the forecasted year:

Plastic price: The supply and demand model determines price and quantity sold in the market. This 
means that if plastic prices increase the clients will ask for lower quantities. The expert knows that the trend of the demand is going to change because the raw material price is going to increase from January 2007 due to new framework contracts. It corresponds to a trend change factor.

Special offer: The company $\mathrm{X}$ wants to reduce stocks in the beginning of the year 2007. A special offer to their clients is then planned to be launched consisting of delivering the double quantity with lower price. The consequence of this factor is that a big part of the demand of January will be transferred to February and March 2007. In this case, this special offer is classified as a transferring factor.

Technical improvements: Nowadays, big companies care about the fulfillment of the environmental demanded requirements. In this frame, the expert predicts that using ecological non-polluting plastic bags is an interesting additional purchase decision criterion for supermarkets. This factor is classified as a jump factor because there would be new clients trying this new concept of plastic bags.

Client situations: Company $\mathrm{X}$ has a client that asks for a fixed quantity of plastic bags per month. This client has announced that its facilities will close during a month after the summer holidays. The consequence of this event will be a decrease in the total monthly demand. However, this client will order new plastic bags for November. This factor is classified as a transient factor.

Table 1 presents the total impact of the different factors on demand per month using the information on the different factors. Figure 5 compares the real 2007 data to the mathematical forecast and to the judgmentally adjusted forecast. The adjusted forecast presents better results in the forecast and percentage error over nine months. Table 2 presents the forecast performance of the proposed approach. The adjusted forecast gives the best results since the MAE has been decreased by $14 \%$ and the MAPE by $18 \%$.

\subsection{Second case study}

In this second application, we are interested in a set of 4 different products provided by a fresh food distributor with historical sales depicted on figure 6. For these different series, the expert identifies that mathematical forecast would not be sufficient to achieve reliable forecast. Different factors due to the customers' demand behavior are considered. The proposed approach is applied and the mathematical forecasts are then adjusted. The deviation between mathematical forecasts and real sales shows the limit of the past data extrapolation to obtain reliable forecast (cf. Fig. 6). By using the structured expert judgement (jump factor for the case on fig 6.a, transient factor on several time periods on fig 6.b and two transient factors on fig 6.c and 6.d), substantial improvements of the forecast accuracy are achieved. Table 3 shows the improvement of the forecast accuracies relying on the error measures of the equations 9 and 10.

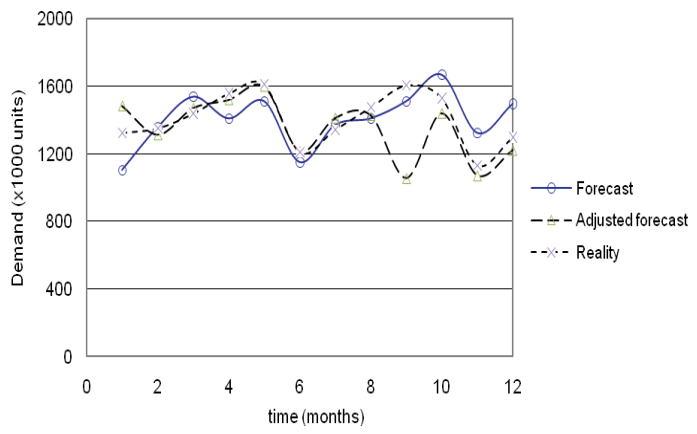

Figure 5. Mathematical forecast, forecast judgmentally adjusted and reality

Table 2. Comparison of the error measures

\begin{tabular}{|c|c|c|}
\hline Measure & MAE & MAPE \\
\hline Math. forecast & 112.2086 & 0.081931 \\
\hline Judg. forecast & 97.54472 & 0.066673 \\
\hline
\end{tabular}

Table 3. Improvement of the forecast accuracy

\begin{tabular}{|c|c|c|c|c|}
\hline Acurracy increase & a & b & c & d \\
\hline MAE & $67 \%$ & $38 \%$ & $77 \%$ & $72 \%$ \\
\hline MAPE & $64 \%$ & $25 \%$ & $20 \%$ & $31 \%$ \\
\hline
\end{tabular}

Table 1. Final monthly adjustments

\begin{tabular}{|c|c|c|c|c|c|}
\hline Month & $\begin{array}{c}\text { Trend change } \\
\text { adjustment }\end{array}$ & $\begin{array}{c}\text { Transferring factor } \\
\text { adjustment }\end{array}$ & $\begin{array}{c}\text { Jump factor } \\
\text { adjustment }\end{array}$ & $\begin{array}{c}\text { Transient factor } \\
\text { adjustment }\end{array}$ & Total adjustment \\
\hline January & -23 & 400 & & & 377 \\
\hline February & -46 & -200 & 200 & & -46 \\
\hline March & -69 & -200 & 200 & & -69 \\
\hline April & -92 & & 200 & & 108 \\
\hline May & -115 & & 200 & & 85 \\
\hline June & -138 & & 200 & & 62 \\
\hline July & -161 & & 200 & & 39 \\
\hline August & -184 & & 200 & & 16 \\
\hline September & -207 & & & -250 & -457 \\
\hline October & -230 & & & & -230 \\
\hline November & -253 & & & & -253 \\
\hline December & -276 & & & & -276 \\
\hline
\end{tabular}



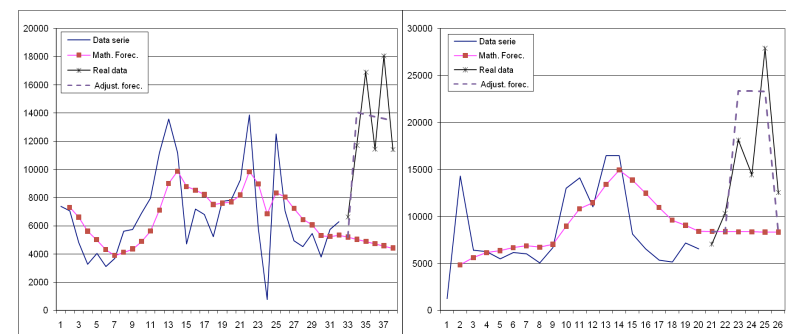

a.

b.

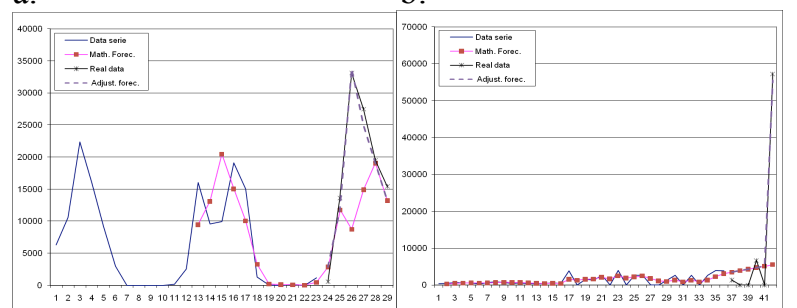

c.

d.

Figure 6. Mathematical forecast, mathematical forecast judgmentally adjusted and reality

\section{CONCLUSION}

We propose a factor-based approach to assist the forecaster, structure his judgment when adjusting forecasts and to enhance the accuracy of global forecasts. Therefore, the forecaster is able to structure and communicate his knowledge regarding future market modification and non-periodic events. It allows forecasters to identify different factors, in order to integrate these events as well as their impacts with mathematical models into global and reliable forecasts. For both presented industrial case studies, the proposed approach has a substantial impact on the accuracy of the resulting forecasts. In fact, by applying our approach, the MAE and the MAPE have been both decreased.

Forecasts are logically improved since the achieved adjustments are based on the expert knowledge. However, if the adjustments are done globally, without structuring or without real analysis of their impacts, their durations and their locations in time, the deviation and the error might increase.

The developed approach is simple and complete, thus allowing the expert to transcribe each possible situation. It presents interesting perspectives concerning the analysis of experts' perceptions, in particular the cognitive process of impact estimation for each considered factor, and their integration into the forecasting process; different experts could have different perceptions of factors, leading to a range of different evaluations for a same factor.

In addition, it is interesting to investigate the evolution of forecast accuracy if experts get different feed-backs based on the comparison between adjusted forecasts and past real demand and occurred events.

\section{REFERENCES}

[1] Makridakis S. and Hyndmann R.J., "Forecasting, methods and applications". John Wiley \& Sons, Inc. Third Edition, pp185-240, 1998.

[2] Lawrence M., Goodwin P., O'connor P. and Önkal D., "Judgmental forecasting: A review of progress over the last 25years", International Journal of Forecasting, Vol. 22, pp493-518, 2006.

[3] Meunier Martins S., Cheikhrouhou N. and Glardon R., "Strategic analysis of products related to the integration of human judgment into demand forecasting", Integrating Human Aspects in Production Management. pp157-173, Springer, New York, 2005.

[4] Sanders N.R. and Ritzman L.P, "Integrating judgmental and quantitative forecasts: Methodologies for pooling marketing and operations information", International Journal of Operations \& Production Management, Vol. 24, No. 5, pp514-529, 2004.

[5] Webby R. and O'connor M., "Judgemental and statistical time series forecasting: a review of the literature", International Journal of Forecasting, Vol. 12, No. 1, pp91-118, 1996.

[6] Bunn D. and Wright G., "Interaction of judgmental and statistical forecasting methods, issues and analysis", Management Science, Vol. 37, No. 5, pp501-18, 1991.

[7] Fildes R., Goodwin P., "Good and bad judgement in forecasting: Lessons from four companies", Foresight, Vol. 8, pp5-10, 2007.

[8] Sanders N.R. and Ritzman L.P, "Bringing judgment into combination forecasts", Journal of Operations Management, Vol. 13, No. 4, pp311-321, 1995.

[9] Edmundson R. H., "Decomposition; a strategy for judgemental forecasting”, Journal of Forecasting, Vol. 9, No. 4, pp305-314, 1990.

[10] Flores B.E., Olson D.L., Wolfe C., "Judgmental adjustment of forecasts: A comparison of methods", International Journal of Forecasting, Vol. 7, No. 4, pp421-433, 1992.

[11] Lee J.K., Oh S.B. and Shin J.C., "UNIK-FCST: Knowledge-Assisted Adjustment of Statistical Forecasts". Expert Systems With Applications, Vol. 1, pp39-49, 1990.

[12] Lee J.K. and Yum C.S., "Judgmental adjustment in time series forecasting using neural networks", Decision Support Systems, Vol.22, pp135-154, 1998.

[13] Armstrong J.S., "Judgmental Bootstrapping: Inferring Experts' Rules for Forecasting”, In J.S. Armstrong (Ed.), Principles of forecasting, pp171-192, 2001. 